\title{
Caracterização comportamental, eletroencefalográfica, eletromiográfica e hematológica resultante da intoxicação por Thevetia peruviana
}

\author{
Adrielle Thais Monteiro dos Santos ${ }^{\mathrm{i}}$
}

\section{Registro DOI: http://dx.doi.org/10.22280/revintervol11ed2.350}

\section{Resumo}

Thevetia peruviana é uma planta nativa da América Central e do Sul, comum em muitas regiões brasileiras e em locais de fácil acesso. Ela tem sido a causa de muitas intoxicações confirmadas pelo ministério da saúde e pelo sistema de informações tóxico farmacológicas. O objetivo deste trabalho é conhecer e caracterizar os padrões eletroencefalográficos e eletromiográficos obtidos após a aplicação do extrato etanólico de Thevetia peruviana em ratos, comprovando efeitos de excitabilidade cardíaca e quimioconvulsivantes. Foram utilizados para os primeiros testes experimentais 27 ratos machos adultos da linhagem Wistar, pesando entre 200 e 250 gramas. Para uma caracterização comportamental da exposição ao extrato, foram utilizados 09 ratos que receberam por via intraperitoneal o extrato etanólico numa dose de $150 \mathrm{mg} / \mathrm{kg}$. A avaliação comportamental da administração do extrato da Thevetia peruviana revelou a ação tóxica da planta. Os registros eletroencefalográficos demonstraram uma diminuição nas forças cerebrais de forma significativa. Conclui-se que a Thevetia tem alto poder de intoxicação e causa entre seus principais efeitos miorrelaxamento e efeito depressor da atividade cerebral.

Palavras-chave: Thevetia peruviana. Intoxicação. Miorrelaxamento.

\section{Behavioral, electrophageophalographic, electromatographic and hematological characterization from intoxication by the peruvian thevetia}

\begin{abstract}
Thevetia peruviana is a native plant from South and Central America, founded in different Brazilian areas, including easy access places. This plant has been associated with several intoxication cases, confirmed by the Ministry of Health and the toxic pharmacologic information system. The present study aims to identify and characterize electroencephalographic and electromyographic patterns presented after the injection of Thevetia peruvianaethanolic extract in rats, proving cardiac excitability and chemoconvulsants effects. The first experimental tests were developed with 27 male wistars, weighing between 200 and 250 grams. In order to evaluate the plant toxicity and behavioral changes, $150 \mathrm{mg} / \mathrm{kg}$ of the plant extract was injected in the intraperitoneal cavity. Toxicity was proven and the electroencephalographic records showed a significant decrease in brain forces. We concluded that Thevetia hold a strong intoxication power, triggering myorelaxation and depression of brain activity as main effects.
\end{abstract}

Keywords: Thevetia peruviana. Intoxication. Myorelaxation. 


\section{Revinter}

Recebido em 06/10/2017 Aceito em 03/05/2018

\section{INTRODUÇÃO}

As intoxicações por plantas têm se tornado um problema bem frequente em todo o mundo, sendo uma ameaça tanto para a saúde dos seres humanos como para os animais que também podem ingerir essas plantas.

O uso de plantas para a ornamentação de parques e jardins é abundante e esconde o perigo de serem tóxicas, pois muitas delas não possuem todos os seus efeitos sobre o organismo elucidados.

Haraguchi (2003) define planta tóxica como "todo vegetal que, introduzido no organismo humano ou de animais domésticos, em condições naturais, é capaz de causar danos que se refletem na saúde e na vitalidade desses seres". Um exemplo de planta tóxica é a Thevetia peruviana, uma planta nativa da América Central e do Sul, pertencente à família Apocynaceae (MANAGIT; SAKURAI; SAIKI, 2017).

Conhecida popularmente pelos nomes: chapéu-de-napoleão, jorro-jorro e bolsa-de-pastor (FRANCO, 2011). Também pode ser identificada por seus sinônimos: Thevetia neriifolia, Cascabela thevetia e Cerbera thevetia. É uma pequena planta perene de 2-6 pés de altura com ampla distribuição geográfica e ecológica e suas partes são utilizadas para fins medicinais em sistemas indianos de medicina (SAMANTA; BHATTACHARYA; RANA, 2016).

No Brasil pode ser encontrada em várias cidades, como Belém- PA (VASCONCELOS et al., 2009), Teresina-PI (SILVA, 2009), Gurupi-TO (TEIXEIRA et al, 2013), Quirinópolis- GO (BASTIEL et al., 2009) e Santiago-RS (SOUZA et al., 2011). Em algumas cidades é muito comum ver a planta cultivada nas calçadas (ZIBBU; BATRA, 2010) devido às suas belas flores.

Segundo o Sistema Nacional de Informações Tóxico-Farmacológicas - SINITOX (2001), $a$ Thevetia peruviana está entre as 16 plantas mais tóxicas, além disso a cada dez casos de intoxicação por plantas, seis ocorrem em crianças menores de dez anos (ASSIS et al, 2009). Mulitos desses casos conhecidos de intoxicação são intencionais e acidentais de humanos através da ingestão de frutas e folhas. Considerando cerca de 10 frutas consumida pode ser fatal para um adulto, e uma única fruta pode ser letal para uma criança (RAMOS-SILVA, 2017).

Apesar de todas as partes da planta serem tóxicas (MEDEIROS; PEREIRA, 2008) a maioria dos registros de intoxicações são devidos a ingestão acidental de sementes, que se localizam no interior do fruto produzido pela Thevetia, principalmente por crianças ( LANGDORF, 1996) 
O poder tóxico da Thevetia peruviana está ligado a substâncias presentes nos tecidos, como a Taventina A, Taventina B, Tevefolina acetilneriifolia, Peruvosida e Nerifolina (HANSSAN et al., 2011; FERNÁNDEZ, 2004; DARREN et al, 2006) esses compostos estão relacionados com os principais sintomas da intoxicação pela Thevetia, que são alterações gastrointestinais (FERNÁNDEZ, 2004; TEIXEIRA et al, 2013; DURASNEL et al, 2014) devido ao bloqueio da $\mathrm{Na}^{+}$e $\mathrm{K}^{+}$ATPase intestinal (ROBERTS et al., 2006).

A ingestão de sementes resulta em um quadro clínico semelhante ao de overdose de digoxina (EDDLESTON, 2000) e seus efeitos colaterais também são semelhantes à intoxicação por digitálicos e se resumem em: dor/queimação, sialorréia, náuseas, vômitos, cólicas abdominais, diarréia. Manifestações neurológicas com cefaléia, tonturas, confusão mental e distúrbios visuais. Distúrbios cardiovasculares: arritmias, bradicardia, hipotensão (SECRETÁRIA DA SÁUDE, 2017).

Os glicosídeos cardíacos desta planta, principalmente a Tavetina A e B, provocam complicações cardíacas as quais frequentemente resultam em bradiarritmias e taquiarritmias, já as substâncias neurotóxicas possuem efeitos neuromusculares, como tremores corporais e movimentos clônicos; neuromotores, como paralisia do pescoço; e psicomotores, como distúrbios e confusão mental. (RUBÉN et al, 2014; OJI; OKAFOR, 1999). Tal estudo corrobora com os sintomas descritos por outros autores, como delírio, dores de cabeça, midríase, convulsões (FERNÁNDEZ, 2004; GONZÁLEZ et al., 2003; TEIXEIRA et al., 2013).

\section{JUSTIFICATIVA}

Por sua presença frequente em jardins e parques e por seu alto poder tóxico, o estudo das intoxicações por Thevetia peruviana é altamente necessário. Sendo uma das que mais causam intoxicação em crianças e animais. Ao aprimorar nosso conhecimento será possível mapear o quadro de intoxicação para poder dar suporte ao tratamento da paciente intoxicado a fim de viabilizar antídotos para o quadro. Assim, com o presente estudo o desenvolvimento e caracterização de novos modelos dos efeitos dessa planta poderão ser analisados, bem como os quadros de intoxicação para obter um tratamento eficaz e viável.

A iniciativa deste estudo é conhecer e caracterizar os padrões eletroencefalográficos e eletromiográficos obtidos após a aplicação do extrato etanólico da Thevetia peruviana (EETP) em ratos, a fim de comprovar a mesma como uma planta que possui substâncias de excitabilidade cardíaca e químioconvulsivantes. O seu efeito mais prevalente são os distúrbios gastrointestinais em 


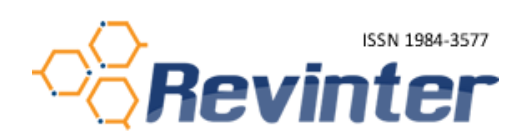

consequência da ingestão. Por ter efeitos estimuladores e depressores sobre o SNC mimetizando as formas de transtornos psiquiátricos encontrados em seres humanos, os modelos animais são responsáveis pelo grande aumento do conhecimento das convulsões e de seu tratamento.

\section{OBJETIVOS}

Objetivo geral:

Analisar os parâmetros comportamentais e eletroencefalográficos de animais submetidos à ingestão de extrato etanólico de Thevetia peruviana. A partir disso, será possível encontrar métodos mais eficientes para tratar indivíduos intoxicados pela planta.

Objetivos específicos:

- Analisar as principais alterações comportamentais dos animais submetidos à dose de $150 \mathrm{mg} / \mathrm{kg}$ por via oral de extrato etanólico de Thevetia peruviana. Verificar-se-á a latência para o aparecimento de cada característica comportamental, bem como sua repetição em cada animal do grupo experimental.

- Caracterizar o eletroencefalograma dos animais submetidos a administração de EETP.

\section{MATERIAIS E MÉTODOS:}

Animais

Foram utilizados para os primeiros testes experimentais 27 ratos machos adultos da linhagem Wistar, pesando entre 200 e 250 gramas. Os animais foram adquiridos no Biotério Central da Universidade Federal do Pará e, durante o experimento, mantidos 3 destes por gaiola no Biotério de Experimentação do Laboratório de Farmacologia e Toxicologia de Produtos Naturais, sob temperatura regulada entre $25^{\circ}$ e $28^{\circ} \mathrm{C}$ e ciclo claro-escuro de 12 horas. Neste período, os animais receberam água e alimentação ad libitum adequados. Todo o experimento foi feito com a aprovação do CEUA, sob registro 99-2015.

Extrato do material botânico 
O material botânico foi coletado na cidade de Belém/PA, e encaminhado para o Laboratório de Farmacologia e Toxicologia de Produtos Naturais para obtenção do extrato etanólico. Após a obtenção do extrato o mesmo foi seco em estufa e diluído a uma concentração de $10 \%$.

\section{Caracterização comportamental}

Para a caracterização comportamental da exposição ao extrato foram utilizados 09 ratos. Os animais receberam por via intraperitoneal (IP), o extrato etanólico numa dose de $150 \mathrm{mg} / \mathrm{kg}$. Após a aplicação os animais ficaram sob observação por 02 horas. Foram observadas as manifestações dos principais efeitos, bem como o seu período de latência, a fim de posteriormente caracterizarmos o quadro de intoxicação por via oral pela planta.

Implante do eletrodo

Os animais foram anestesiados com uma associação de Xilazina $(5 \mathrm{mg} / \mathrm{kg})$ e quetamina $(50 \mathrm{mg} / \mathrm{kg})$ por via i.p. Após o animal apresentar perda de reflexo interdigital foi fixado no aparelho esteriotáxico, para a implantação de eletrodos. O procedimento foi iniciado com a tricotomia da pele da região da cabeça, com adequada assepsia, foi feito a infiltração de lidocaína a $2 \%$ no local da incisão cirúrgica que contem $3 \mathrm{~cm}$ rostro-caudal na linha mediana, expondo a superfície óssea do crânio. A lidocaína também foi aplicada no conduto auditivo externo para reduzir os efeitos da pressão das barras do aparelho esteriotáxico. Após a exposição do osso do crânio foram feitas duas perfurações com aparelho odontológico, obedecendo às coordenadas esteriotáxicas $0,96 \mathrm{~mm}$ posterior para bregma, $2 \mathrm{~mm}$ latero-lateral, onde foram implantados os eletrodos que apresentam $1 \mathrm{~mm}$ de diâmetro e acomodados na Dura-máter, e fixado com acrílico autopolimerizante de uso odontológico.

\section{Delineamento Experimental}

Após o procedimento cirúrgico os animais foram mantidos 1 por caixa e acondicionados no biotério de experimentação. Divididos em dois grupos de 9 animais (controle e tratado com EETP). No sétimo dia após a cirurgia, os eletrodos foram conectados a um sistema de aquisição de dados, composto por um amplificador de alta impedância (Grass Technologies, P511), monitorado por um osciloscópio (Protek, 6510). Os dados foram continuamente digitalizados em uma taxa de $1 \mathrm{KHz}$ por um computador equipado com uma placa de aquisição de dados (National Instruments, Austin, TX) e estes armazenados em disco rígido e, processados através de um software especializado (LabVIEW express). O eletrodo de registro estava localizado no lado direito do hemisfério, e do lado esquerdo, o eletrodo de referência. 
Após 10 minutos de acomodação, foi feito a aplicação da droga por via intraperitoneal na dose de $150 \mathrm{mg} / \mathrm{kg}$. A partir desse momento será medido o tempo de latência para a ocorrência na mudança de traçado e o total de tempo para cada registro será de 60 minutos.

\section{Análise Estática}

Os dados foram analisados através das médias e desvio padrão. A comparação entre os dados foi feita usando um software Graphpad Prism ${ }^{\circledR}$ 5.0, através do teste de ANOVA não paramétricos, não pareados seguida do teste de Tukey.

\section{RESULTADOS:}

Resultados da Análise Comportamental

A avaliação comportamental da administração do extrato etanólico da Thevetia peruviana revelou a ação tóxica da planta. Foram observados seis padrões comportamentais com períodos de latência próprios. As características comportamentais, por ordem de aparecimento foram: Imobilidade, Eriçamento de vibrissas, Miorrelaxamento sem perda do reflexo de postura, Respiração costo-abdominal, Miorrelaxamento com a perda do reflexo de postura e Respiração agônica (Abdômino-costal).

\begin{tabular}{c|c}
\hline $\begin{array}{c}\text { Característica } \\
\text { comportamental }\end{array}$ & Latência em segundos \\
\hline
\end{tabular}

Imobilidade

$$
220 \pm 45
$$

Eriçamento de vibrissas

$$
262 \pm 35
$$

\section{Miorrelaxamento sem perda} do reflexo de postura.

$293 \pm 52$

\begin{tabular}{ll}
\hline $\begin{array}{c}\text { Respiração } \\
\text { abdominal. }\end{array}$ & costo- \\
\end{tabular}

Revinter, v. 11, n. 02, p. 99-113, jun. 2018. 
Miorrelaxamento com perda

do reflexo de postura.

$$
380 \pm 62
$$

Respiração agônica

característica abdômino-costal

$402 \pm 93$

Tabela 01-Características comportamentais de acordo com o tempo de latência.

\section{Resultados da Análise eletroencefalográfica}

Os registros eletroencefalográficos demonstraram uma diminuição nas forças cerebrais de forma significativa, evidenciada no gráfico de distribuição de frequências, pode ser observado na faixa de frequências até $50 \mathrm{HZ}$ (fig1. A). A distribuição linear de frequência demonstrou que a amplitude para os animais tratados foi de $3,89 \pm 0,2627 \mathrm{mV}^{2} / \mathrm{Hz} \times 10^{-3}$, demonstrou diferença estatística para o grupo basal $8,548 \pm 2,432 \mathrm{mV}^{2} / \mathrm{Hz} \times 10^{-3}$. Os traçados dos registros eletroencefalográficos demonstraram diferença na amplitude do sinal captado pelo eletrodo, a distribuição de energia no espectrograma demonstrou menor distribuição de frequência para os animais tratados (fig. D e F).

A

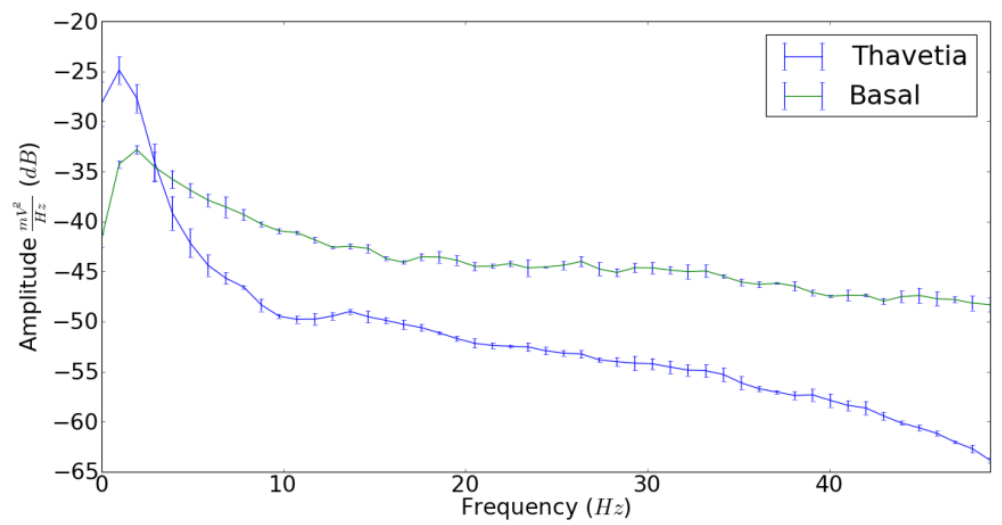

B

Revinter, v. 11, n. 02, p. 99-113, jun. 2018. 


\section{Revinter}

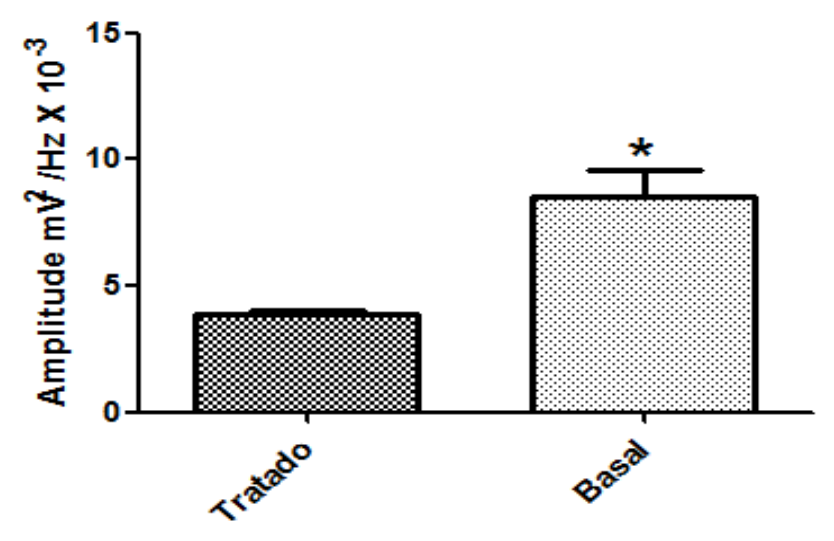

C

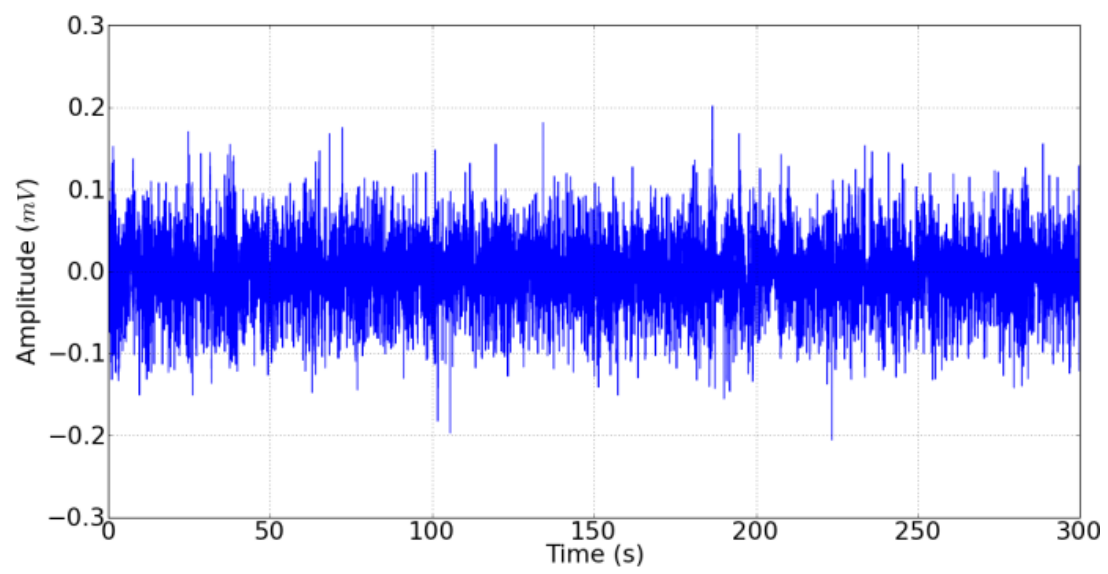

D

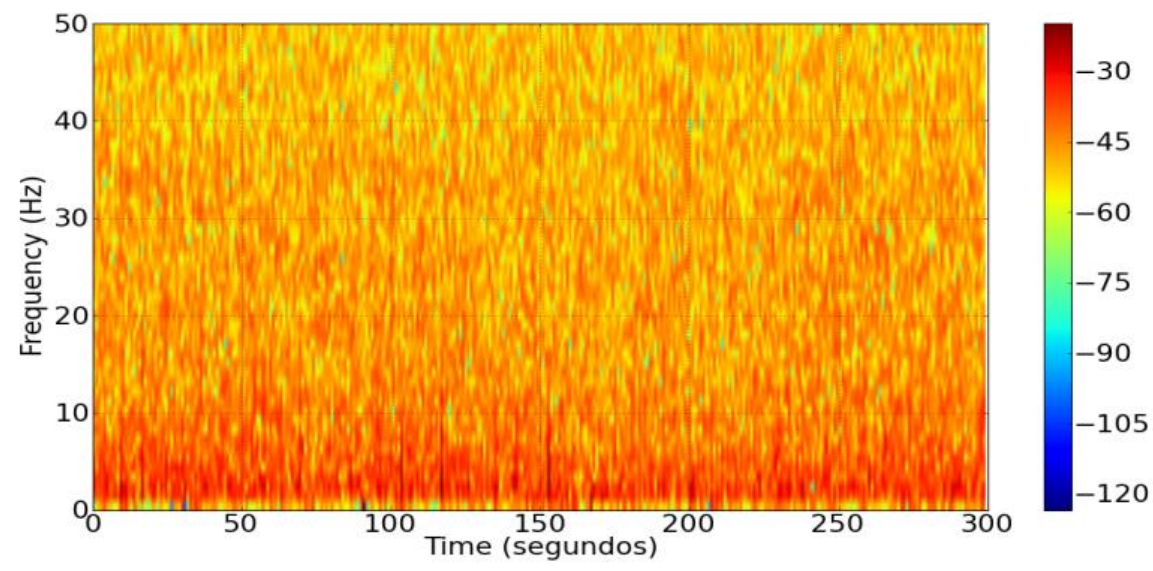

Revinter, v. 11, n. 02, p. 99-113, jun. 2018. 


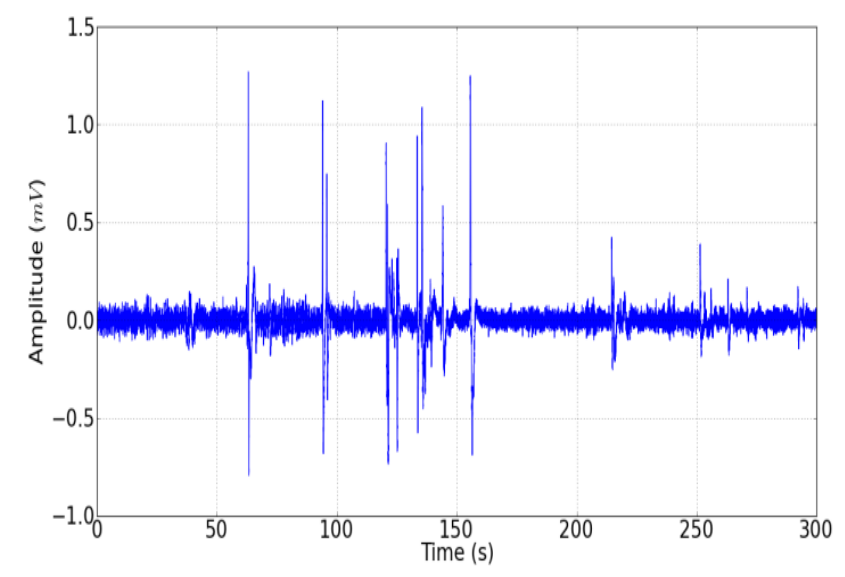

$\mathbf{F}$

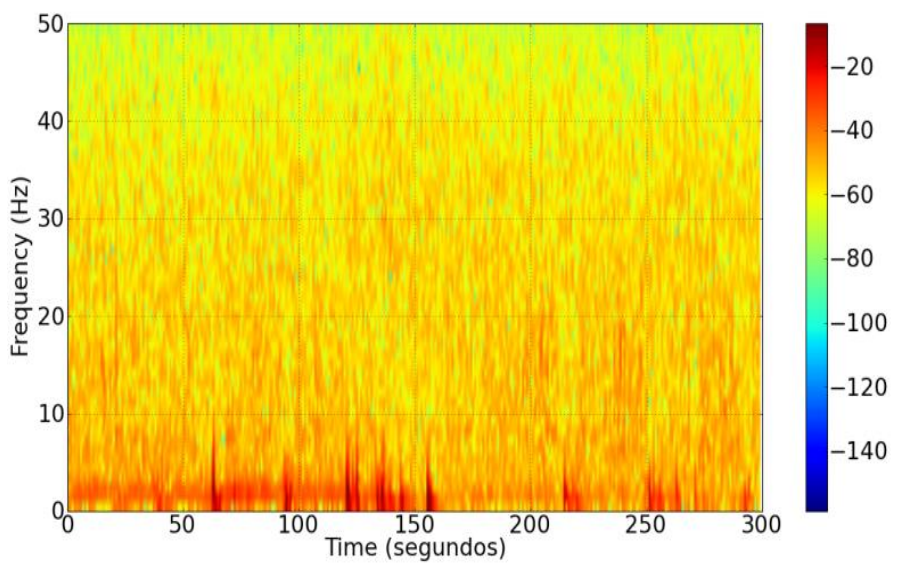

Figura 1. Registro eletrofisiológico e gráfico de potência até $50 \mathrm{~Hz}$. Gráfico de distribuição de frequência em escala logarítmica (A), Gráfico de potência em escala linear (B), Traçados eletrofisiológicos e espectrograma no estado Basal (C) e (D), traçado eletrofisiológico após a aplicação do EET (E) e (F).

O registro eletroencefalográfico foi desmembrado e as forças cerebrais Theta e Gamma analisadas de forma independente. A distribuição linear de frequência na força Theta, demonstrou que a amplitude para os animais tratados foi de $0,1410 \pm 0,04369 \mathrm{mV}^{2} / \mathrm{Hz} \times 10^{-3}$, que foi diferente estatisticamente do grupo basal $0,8560 \pm 0,04092 \mathrm{mV}^{2} / \mathrm{Hz} \times 10^{-3}$. A distribuição de energia no espectrograma demonstrou maior intensidade de frequência para os animais no estado basal, Revinter, v. 11, n. 02, p. 99-113, jun. 2018. 
demonstrando que a aplicação do EET, provocou menor atividade cerebral nas frequências mais Baixas( fig 2. B e C).

A

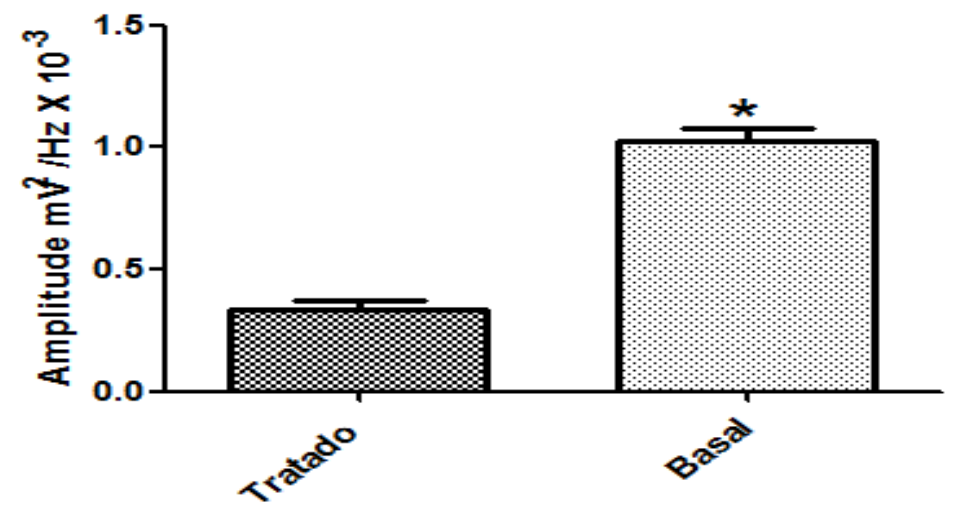

B

BASAL

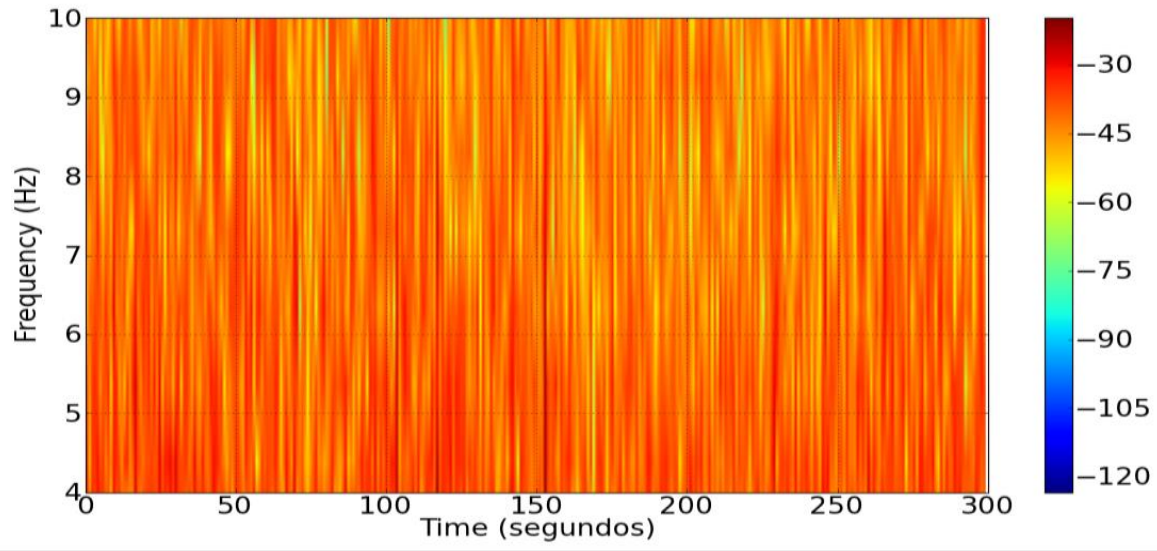


C

\section{Revinter}

TRATADO

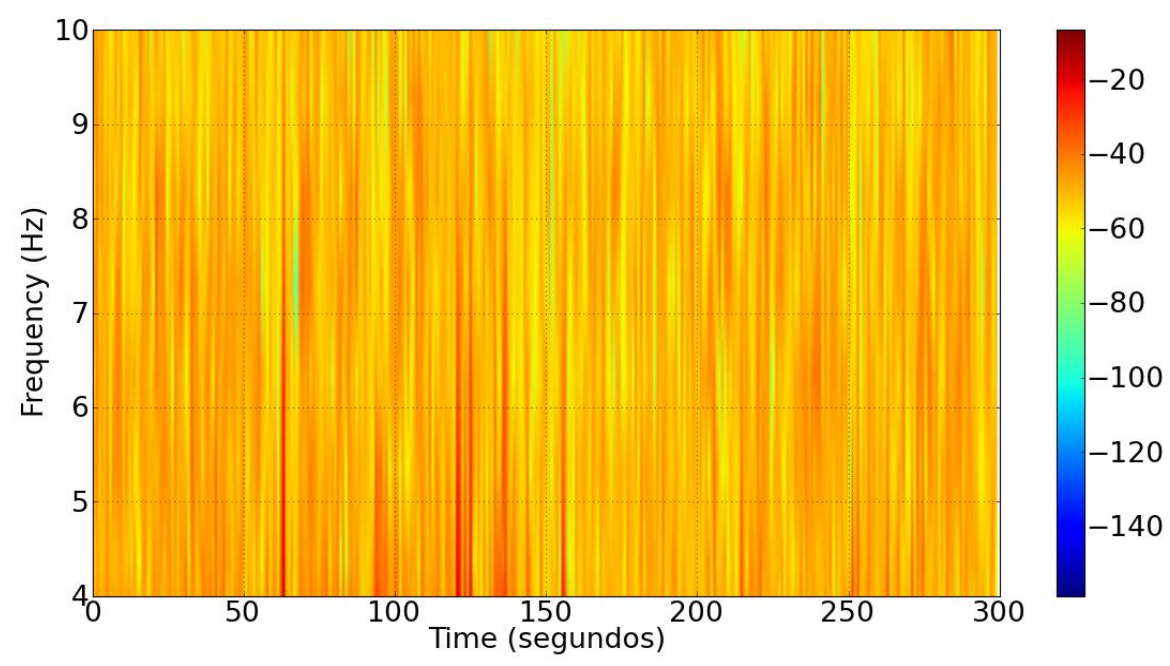

Figura 3. Distribuição de potência linear e Gamma $(20-50 \mathrm{~Hz})$ (A), e respectivos espectrogramas Basal (B) e Tratado (C).

Na força Gamma a distribuição linear de frequência demonstrou uma amplitude, para os animais tratados de $0,3364 \pm 0,08082 \mathrm{mV}^{2} / \mathrm{Hz} \times 10^{-3}$, com diferença estatística para o grupo basal $1,029 \pm 0,1249 \mathrm{mV}^{2} / \mathrm{Hz} \times 10^{-3}$. A distribuição de energia no espectrograma demonstrou, também, uma maior intensidade de frequência para os animais no estado basal, demonstrando que a aplicação do EET, similar ao ocorrido para Theta, provocou menor atividade cerebral nas frequências de maior intensidade como Gamma (fig 3. B e C).

Os resultados evidenciam de forma uma redução da atividade do sistema nervoso central na dose estudada e em ambas avaliações: comportamental e eletrofisiológica. É preciso salientar que não há na literatura trabalhos que evidencie de forma complementar com a eletrofisiologia esse tipo de característica. Outros autores utilizando modelos animais diferentes, doses diferentes e tipo de obtenção do extrato diferente já caracterizaram comportamentalmente a intoxicação por Thevetia peruviana.

\section{CONCLUSÃO}

Os dados obtidos revelaram que a Thevetia tem alto poder de intoxicação e causa entre seus efeitos miorrelaxamento e efeito depressor da atividade cerebral. Desta forma as evidências indicam 
que em estudos futuros o isolamento dessas substâncias podem ter utilidade terapêutica se durante a utilização demonstrarem reversibilidade dos seus efeitos.

Existe a necessidade de se estudar mais a fundo os efeitos da planta em questão, apenas assim caracterizando seus efeitos e seus princípios ativos poderemos utilizá-la de forma correta.

\section{REFERÊNCIAS BIBLIOGRÁFICAS}

ASSIS, M.a et al. Presença de plantas tóxicas em escolas públicas do município do Rio de Janeiro: dados preliminares. 2009. Disponível em: <https://www.arca.fiocruz.br/handle/icict/475>. Acesso em: 10.08 .2017

BATISTEL, L.M et al. Diagnóstico qualitativo e quantitativo da arborização urbana nos bairros Promissão e Pedro Cardoso, Quirinópolis, Goiás. Revsbau, Piracicaba, v. 4, n. 3, p.110-129, 2009.

BOCHNER, R. et al. Problemas associados ao uso de plantas medicinais comercializadas no Mercadão de Madureira, município do Rio de Janeiro, Brasil. Rev. Bras. PI. Med., Botucatu, v. 14, n. 3, p.537-547, 2012.

COSTA, F.M. et al. Anais Do IX Congresso De Ecologia Do Brasil, 2009, São Lourenço. Riscos De Intoxicação De Animais Na Pecuária, Por Plantas Tóxicas: Uma Revisao Bibliográfica. Minas Gerais: SEB, 2009. p. Disponível em: <http://www.sebecologia.org.br/2009/resumos_ixceb/84.pdf>. Acesso em: 01 ago. 2017.

DURASNEL, P. et al. Intoxications graves lors de treitements traditionnels par les plantes à Mayotte. Société de pathologie exotique et Lavoisier, n. 107, p. 306-311, jun. 2014.

EDDLESTON, M. et al. Acute yellow oleander (Thevetia peruviana) poisoning: cardiac arrhythmias, electrolyte disturbances, and serum cardiac glycoside concentrations on presentation to hospital. Heart, v. 83, n. 3, p. 301-306, 2000.

FRANCO, M. Chapéu-de-Napoleão (Thevetia peruviana). 2011. Disponível em: <https://plantasornamentais.blogspot.com.br/2011/04/chapeu-de-napoleao-thevetia-peruviana.html>. Acesso em: 03 jul. 2017. 
GARCÍA, C.; LUNA, Z. Intoxicación por Thevetia peruviana (hueso o codo de fraile). Presentación de un caso. Revista de la Associación Mexicana de Medicina Crítica y Terapia Intensiva, v. XXVII, n. 4, p. 245-248, out/dez. 2003.

GONZÁLEZ, Y. A. de et al. Intoxicación por la administración de tabletas de Thevetia peruviana como tratamiento para bajar de peso: presentación de un caso. Revista de Toxicología, v. 20, n. 3, p. 221-223, 2003.

HASSAN, M.M. et al. Studies on the antidiarrhoeal, antimicrobial and cytotoxic activities of ethanol-extracted leaves of yellow oleander (Thevetia peruviana). Open Veterinary Journal, V. 1, p. 28-31, fev. 2011.

HARAGUCHI, M. Plantas tóxicas de interesse na pecuária. Revista O Biológico., 65: 37 - 39, 2003.

KARERU, P.G et al. Anti-termite and antimicrobial properties of paint made from Thevetia peruviana (Pers.) Schum. Oil extract. African Journal of Pharmacy and Pharmacology, V. 4, P. 087-089, fev. 2010.

LANGDORF, S.D.; Boor, P.J. Oleander toxicity: na examination of human and toxic exposures. Toxicology, v.109, n. s/n, p 1-13, 1996.

MAGALHÃES, B; ALMEIDA, M.P de. Crescimento e desenvolvimento de mudas de Thevetia neriifolia L. em casa de vegetação. 2014.

MANAGIT, C.; SAKURAI, H.; SAIKI, I. Ethanolic extract of Thevetia peruviana flowers enhances TNF- $\alpha$ and TRAIL-induced apoptosis of human cervical cancer cells via intrinsic and extrinsic pathways. Oncology Letters, v. 13, n. 4, p. 2791-2798, 2017

MEDEIROS, Luiz Fernando Souza; PEREIRA, Marcio. Espécies com princípios tóxicos, empregados na arborização urbana do bairro Nossa Senhora das Graças-Miguelópolis, SP. Nucleus, v. 5, n. 2, 2008.

MOURA, M.D.B de; AGRA, M.F. Apocynaceae tóxicas e medicinais ocorrentes nos estados de pernambuco e paraíba, brasil. Acta Boto Bras, Recife, v. 2, n. 3, p.273-279, 1989.

OJI, O.; OKAFOR, Q. Toxicological Studies on Stem Bark, Leaf and Seed Kernel of Yellow Oleander (Thevetia peruviana). Phytotherapy Research, v. 14, p. 133-135, jun. 1999.

OLIVER-BEVER, B. Medicinal Plants in Tropical West Africa. Cambridge University Press, p. $366,1986$. 
PANIGRAHI, A.; RAUT, S. K. Thevetia peruviana (Family: Apocynaceae) in the control of slug and snail pests. Mem. Inst. Oswaldo Cruz [online], v.89, n.2, p. 247-250, 1994.

RAMOS-SILVA, A. et al. Anticancer potential of Thevetia peruviana fruit methanolic extract. BMC complementary and alternative medicine, v. 17, n. 1, p. 241, 2017.

ROBERTS, D. M. et al. Pharmacokinetics of digoxin cross-reacting substances in patients with acute yellow oleander (Thevetia peruviana) poisoning, including the effect of activated charcoal. Ther Drug Mo, v. 28, n. 6, p. 784, 2006

SAMANTA, J.; BHATTACHARYA, S.; RANA, A. C. Antifertility activity of Thevetia peruviana (Pers.) K. Schum leaf in female Sprague-Dawley rat. Indian journal of pharmacology, v. 48, n. 6, p. 669, 2016.

Secretaria da Saúde (Org.). Intoxicações por Plantas Tóxicas. Disponível em:

<http://www.saude.pr.gov.br/arquivos/File/zoonoses_intoxicacoes/plantas/Intoxicacoes_por_Pla ntas_Toxicas.pdf >. Acesso em: 01 ago. 2017.

SILVA, L.C. Plantas ornamentais tóxicas presentes no shopping riverside walk em Teresina - PI. Revsbau, Piracicaba, v. 4, n. 3, p.69-85, 2009.

SINITOX/CICT/FIOCRUZ; CIT/PA - Belém; CIAVE/BA -Salvador; CCI/SP - São Paulo; CCI/SP - Campinas; CIAVE/ MT - Cuiabá; CIT/RS - Porto Alegre. Plantas Tóxicas no Brasil. Julho, 2001 (cartaz e folder)

SOUZA, A.R.C de et al. Identificação das espécies ornamentais nocivas na arborização urbana de Santiago/RS. Revsbau, Piracicaba, v. 6, n. 2, p.44-57, 2011

TEIXEIRA, L. et al. Aspectos Toxicológicos de Thevetia peruviana e perfil dos usuários em Gurupi-TO. Revista Científica do ITPAC, Araguaina, v.6, n.4, Pub.4, out. 2013.

VASCONCELOS, J.; VIEIRA, J.; VIEIRA, E. Plantas Tóxicas: Conhecer para Prevenir. Revista Científica da UFPA, V.7, n. 01, p. 01-10, 2009.

YADAV, S.; SINGH, S.P.; MITTAL, P.K. Toxicity of Thevetia peruviana (yellow oleander) against larvae of Anopheles stephensi and Aedes aegypti vectors of malaria and dengue. Journal of Entomology and Zoology Studies V.1, n.6, p. 85-87, nov. 2013. 


\section{Revinter}

ZAMANI, M. D.; ASLANI, M.D. Cardiac Findings in Acute Yellow Oleander Poisoning. Journal of cardiovascular disease research, v. 1, n.1, p. 27-28, jan./mar. 2010.

ZIBBU, G.; BATRA, A. Effect of adeninesulphate on organogenesis via leaf culture in an ornamental plant: Thevetia peruviana (pers.) Schum. International Journal Of Pharma And Bio Sciences, India, v. 1, n. 2, p.1-9, 2010.

\footnotetext{
' Acadêmica de Biomedicina da Universidade Federal do Pará. Estágio no Laboratório de Farmacologia e Toxicologia de Produtos Naturais (LFTPN-UFPA). E-mail para contato: adriellethaiss@gmail.com
} 